\title{
Numerical Solution of a Quadratic Integral Equation through Classical Schauder Fixed Point Theorem
}

\author{
Merve Temizer Ersoy ${ }^{1 *}$
}

\begin{abstract}
In this paper, we investigate the existence of at least one solution on the closed interval for quadratic integral equations with non-linear modification of the argument in Hölder spaces using the technique in the classical Schauder fixed point theorem.

Keywords: Fredholm integral equation, Hölder condition, Schauder fixed point theorem.

2010 AMS: Primary 45B05, 45G10, 47H10

${ }^{1}$ Department of Mathematics, Faculty of Science and Arts, Kahramanmaraş Sütçü Imam University, Kahramanmaraş, Turkey, ORCID: 0000-0003-4364-9144

Received: 13 December 2021, Accepted: 10 March 2021, Available online: 29 March 2021
\end{abstract}

\section{Introduction}

Integral equations arise naturally in various applications in describing numerous real universe problems. As well, quadratic integral equations have numerous useful applications in describing uncountable events and problems of the real world. For instance, quadratic integral equations are often applicable in the traffic theory, in the theory of radiative transfer, in the theory of neutron transport and kinetic theory of gases. Several authors have comprehensively studied the integral equations and the solution of them in this references $[1,2,3,4,5,6,7,8,9,10,11,12,13,14,15,16,17,18,19,20,21,22,23]$. Moreover, M. Benchohra and M. A. Darwish et al. [1] study the existence of the unique solution, defined on a semi-infinite interval $J:[0, \infty)$ for the following quadratic integral equations with a linear modification of the argument

$$
x(t)=f(t)+(A x)(t) \int_{0}^{T} u(t, s, x(s), x(\alpha s)) d s, t \in J .
$$

where $f: J \rightarrow \mathbb{R}, u: J \times J_{T} \times \mathbb{R}^{2} \rightarrow \mathbb{R}$ are given functions, $0<\alpha<1, J_{T}=[0, T]$ and $A: C(J ; \mathbb{R}) \rightarrow C(J ; \mathbb{R})$ is an appropriate operator. Here $C(J ; \mathbb{R})$ denotes the space of continuous functions $x: J \rightarrow \mathbb{R}$.

This article concerns the entity of solutions of the following a quadratic integral equation of Fredholm type,

$$
x(t)=\left(T_{1} x\right)(t)+\left(T_{2} x\right)(t) \int_{0}^{1} k(t, \tau)\left(T_{3} x\right)(\tau) d \tau, t \in I=[0,1] .
$$

where $k$ is given function, $T_{1}, T_{2}, T_{3}$ are given operators satisfying conditions specified later and $x$ is unknown function.

\section{Preliminaries}

Let $[a, b]$ be a closed interval in $\mathbb{R}$, by $C[a, b]$ we indicate the space of continuous functions defined on $[a, b]$ equipped with the supremum norm, i.e.,

$$
\|x\|_{\infty}=\sup \{|x(t)|: t \in[a, b]\}
$$


for $x \in C[a, b]$. For a fixed $\alpha$ with $0<\alpha \leq 1$, by $H_{\alpha}[a, b]$ we will indicate the spaces of the real functions $x$ defined on $[a, b]$ and satisfying the Hölder condition, that is, those functions $x$ for which there exists a constant $H_{x}^{\alpha}$ such that

$$
|x(t)-x(s)| \leq H_{x}^{\alpha}|t-s|^{\alpha}
$$

for all $t, s \in[a, b]$. It is well proved that $H_{\alpha}[a, b]$ is a linear subspaces of $C[a, b]$. Also, for $x \in H^{\alpha}[a, b]$, by $H_{x}^{\alpha}$ we will indicate the least possible stable for which inequality (2.1) is satisfied. Rather, we put

$$
H_{x}^{\alpha}=\sup \left\{\frac{|x(t)-x(s)|}{|t-s|^{\alpha}}: t, s \in[a, b] \text { and } t \neq s\right\} .
$$

The space $H_{\alpha}[a, b]$ with $0<\alpha \leq 1$ may be equipped with the norm

$$
\|x\|_{\alpha}=|x(a)|+H_{x}^{\alpha}
$$

for $x \in H_{\alpha}[a, b]$. Here, $H_{x}^{\alpha}$ is defined by (2.2). In [2], the authors demonstrated that $\left(H_{\alpha}[a, b],\|\cdot\|_{\alpha}\right)$ with $0<\alpha \leq 1$ is a Banach space.

Lemma 2.1. For $0<\alpha \leq 1$ and $x \in H_{\alpha}[a, b]$, we have:

$$
\|x\|_{\infty} \leq \max \left(1,(b-a)^{\alpha}\right)\|x\|_{\alpha} .
$$

In particular, the inequality $\|x\|_{\infty} \leq\|x\|_{\alpha}$ is satisfied for $a=0$ and $b=1$, [2].

Lemma 2.2. For $0<\alpha<\beta \leq 1$, we have

$$
H_{\beta}[a, b] \subset H_{\alpha}[a, b] \subset C[a, b] .
$$

Furthermore, for $x \in H_{\beta}[a, b]$, we have:

$$
\|x\|_{\alpha} \leq \max \left(1,(b-a)^{\beta-\alpha}\right)\|x\|_{\beta} .
$$

Particularly, the inequality $\|x\|_{\infty} \leq\|x\|_{\alpha} \leq\|x\|_{\beta}$ is satisfied for $a=0$ and $b=1$, [2].

Lemma 2.3. Let's assume that $0<\alpha<\beta \leq 1$ and $E$ is a bounded subset in $H_{\beta}[a, b]$, then $E$ is a relatively compact subset in $H_{\alpha}[a, b],[3]$.

Lemma 2.4. Assume that $0<\alpha<\beta \leq 1$ and by $B_{r}^{\beta}$ we indicate the ball centered at $\theta$ and radius $r$ in the space $H_{\beta}[a, b]$, i.e., $B_{r}^{\beta}=\left\{x \in H_{\beta}[a, b]:\|x\|_{\beta} \leq r\right\}$. Then $B_{r}^{\beta}$ is a closed subset of $H_{\alpha}[a, b]$, [3].

Corollary 2.5. Assume that $0<\alpha<\beta \leq 1$ and $B_{r}^{\beta}=\left\{x \in H_{\beta}[a, b]:\|x\|_{\beta} \leq r\right\}$, then $B_{r}^{\beta}$ is a compact subset in the space $H_{\alpha}[a, b],[3]$.

Theorem 2.6 (Schauder's fixed point theorem). Let $E$ be a nonempty, compact and convex subset of a Banach space $(X,\|\cdot\|)$, convex and let $T: E \rightarrow E$ be a continuity mapping. Then $T$ has at least one fixed point in E, [4].

\section{Main Result}

Theorem 3.1. Assume that the following conditions (i) - (iv) are satisfied:

(i) The operators $T_{1}, T_{2}: H_{\beta}[0,1] \rightarrow H_{\beta}[0,1]$ are continuous on $H_{\beta}[0,1]$ with respect to the norm $\|\cdot\|_{\alpha}$. Also, $T_{1}$ and $T_{2}$ hold the inequalities

$$
\left\|T_{1} x\right\|_{\beta} \leq f_{1}\left(\|x\|_{\beta}\right) \text { and }\left\|T_{2} x\right\|_{\beta} \leq f_{2}\left(\|x\|_{\beta}\right)
$$

for any $x \in H_{\beta}[0,1]$, where $\alpha$ and $\beta$ are the fixed constants satisfying $0<\alpha<\beta \leq 1$ and the functions $f_{1}, f_{2}: \mathbb{R}_{+} \rightarrow \mathbb{R}_{+}$ are nondecreasing on $\mathbb{R}_{+}$.

(ii) $k:[0,1] \times[0,1] \rightarrow \mathbb{R}$ is a continuous function such that there exists a constant $k_{\beta}>0$ satisfying

$$
|k(t, \tau)-k(s, \tau)| \leq k_{\beta}|t-s|^{\beta},
$$

for any $t, s, \tau \in[0,1]$. 
(iii) The operators $T_{3}: H_{\beta}[0,1] \rightarrow C[0,1]$ is continuous on $H_{\beta}[0,1]$ with respect to the norm $\|\cdot\|_{\alpha}$. Also, $T_{3}$ holds the inequality

$$
\left\|T_{3} x\right\|_{\infty} \leq f_{3}\left(\|x\|_{\beta}\right)
$$

for any $x \in H_{\beta}[0,1]$, where $\alpha$ and $\beta$ are the fixed constants satisfying $0<\alpha<\beta \leq 1$ and the functions $f_{3}: \mathbb{R}_{+} \rightarrow \mathbb{R}_{+}$is nondecreasing on $\mathbb{R}_{+}$.

(iv) There exists a positive solution $r_{0}$ of the inequality

$$
f_{1}(r)+\left(2 K+k_{\beta}\right) f_{2}(r) f_{3}(r) \leq r
$$

where the constant $K$ is defined by

$$
\sup \left\{\int_{0}^{1}|k(t, \tau)| d \tau: t \in[0,1]\right\} \leq K .
$$

Then the equation (1.1) has at least one solution $x=x(t)$ belonging to space $H_{\alpha}[0,1]$.

Proof. We take for arbitrarily fixed $t, s \in[0,1],(t \neq s)$ and let us consider $x \in H_{\beta}[0,1]$ and the operator $F$ defined on the space $H_{\beta}[0,1]$ by the formula:

$$
(F x)(t)=\left(T_{1} x\right)(t)+\left(T_{2} x\right)(t) \int_{0}^{1} k(t, \tau)\left(T_{3} x\right)(\tau) d \tau,
$$

for $t \in[0,1]$. Then, in view of our assumptions we get

$$
\begin{aligned}
(F x)(t)-(F x)(s)= & \left(T_{1} x\right)(t)+\left(T_{2} x\right)(t) \int_{0}^{1} k(t, \tau)\left(T_{3} x\right)(\tau) d \tau-\left(T_{1} x\right)(s)-\left(T_{2} x\right)(s) \int_{0}^{1} k(s, \tau)\left(T_{3} x\right)(\tau) d \tau \\
= & \left(T_{1} x\right)(t)-\left(T_{1} x\right)(s)+\left(T_{2} x\right)(t) \int_{0}^{1} k(t, \tau)\left(T_{3} x\right)(\tau) d \tau-\left(T_{2} x\right)(s) \int_{0}^{1} k(s, \tau)\left(T_{3} x\right)(\tau) d \tau \\
& +\left(T_{2} x\right)(s) \int_{0}^{1} k(t, \tau)\left(T_{3} x\right)(\tau) d \tau-\left(T_{2} x\right)(s) \int_{0}^{1} k(t, \tau)\left(T_{3} x\right)(\tau) d \tau \\
= & \left(T_{1} x\right)(t)-\left(T_{1} x\right)(s)+\left(\left(T_{2} x\right)(t)-\left(T_{2} x\right)(s)\right) \int_{0}^{1} k(t, \tau)\left(T_{3} x\right)(\tau) d \tau \\
& +\left(T_{2} x\right)(s) \int_{0}^{1}(k(t, \tau)-k(s, \tau))\left(T_{3} x\right)(\tau) d \tau
\end{aligned}
$$

and

$$
\begin{aligned}
\frac{|(F x)(t)-(F x)(s)|}{|t-s|^{\beta} \leq} & \frac{\left|\left(T_{1} x\right)(t)-\left(T_{1} x\right)(s)\right|}{|t-s|^{\beta}}+\frac{\left|\left(T_{2} x\right)(t)-\left(T_{2} x\right)(s)\right|}{|t-s|^{\beta}} \int_{0}^{1}|k(t, \tau)|\left|\left(T_{3} x\right)(\tau)\right| d \tau \\
& +\frac{\left|\left(T_{2} x\right)(s)\right|}{|t-s|^{\beta}} \int_{0}^{1}|k(t, \tau)-k(s, \tau)|\left|\left(T_{3} x\right)(\tau)\right| d \tau \leq H_{T_{1} x}^{\beta}+\left\|T_{2} x\right\|_{\beta}\left\|T_{3} x\right\|_{\infty} \int_{0}^{1}|k(t, \tau)| d \tau \\
& +\left\|T_{2} x\right\|_{\infty}\left\|T_{3} x\right\|_{\infty} \int_{0}^{1} \frac{|k(t, \tau)-k(s, \tau)|}{|t-s|^{\beta}} d \tau \\
\leq & H_{T_{1} x}^{\beta}+\left\|T_{2} x\right\|_{\beta}\left\|T_{3} x\right\|_{\infty} K+\left\|T_{2} x\right\|_{\beta}\left\|T_{3} x\right\|_{\infty} \int_{0}^{1} k_{\beta} \frac{|t-s|^{\beta}}{|t-s|^{\beta}} d \tau \\
\leq & H_{T_{1} x}^{\beta}+f_{2}\left(\|x\|_{\beta}\right) f_{3}\left(\|x\|_{\beta}\right) K+f_{2}\left(\|x\|_{\beta}\right) f_{3}\left(\|x\|_{\beta}\right) k_{\beta} \\
= & H_{T_{1} x}^{\beta}+\left(K+k_{\beta}\right) f_{2}\left(\|x\|_{\beta}\right) f_{3}\left(\|x\|_{\beta}\right) .
\end{aligned}
$$

This demonstrates that the operator $F$ maps $H_{\beta}[0,1]$ into itself. Besides, for any $x \in H_{\beta}[0,1]$, we get

$$
\begin{aligned}
|(F x)(0)| & \leq\left|\left(T_{1} x\right)(0)\right|+\left|\left(T_{2} x\right)(0)\right| \int_{0}^{1}|k(0, \tau)|\left(T_{3} x\right)(\tau) \mid d \tau \\
& \leq\left|\left(T_{1} x\right)(0)\right|+\left\|T_{2} x\right\|_{\infty}\left\|T_{3} x\right\|_{\infty} K \\
& \leq\left|\left(T_{1} x\right)(0)\right|+\left\|T_{2} x\right\|_{\beta}\left\|T_{3} x\right\|_{\infty} K \\
& \leq\left|\left(T_{1} x\right)(0)\right|+f_{2}\left(\|x\|_{\beta}\right) f_{3}\left(\|x\|_{\beta}\right) K .
\end{aligned}
$$


By the inequalities by (3.1) and (3.2), we derive that

$$
\begin{aligned}
\|F x\|_{\beta} & \leq\left\|T_{1} x\right\|_{\beta}+\left(2 K+k_{\beta}\right) f_{2}\left(\|x\|_{\beta}\right) f_{3}\left(\|x\|_{\beta}\right) \\
& \leq f_{1}\left(\|x\|_{\beta}\right)+\left(2 K+k_{\beta}\right) f_{2}\left(\|x\|_{\beta}\right) f_{3}\left(\|x\|_{\beta}\right) .
\end{aligned}
$$

Since positive number $r_{0}$ is the solution of the inequality given in hypothesis $(i v)$, from (3.3), we conclude that the inequality

$$
\|F x\|_{\beta} \leq f_{1}\left(r_{0}\right)+\left(2 K+k_{\beta}\right) f_{2}\left(r_{0}\right) f_{3}\left(r_{0}\right) \leq r_{0}
$$

holds. As a results, it follows that $F$ transforms the ball

$$
B_{r_{0}}^{\beta}=\left\{x \in H_{\beta}[0,1]:\|x\|_{\beta} \leq r_{0}\right\}
$$

into itself. That is, $F: B_{r_{0}}^{\beta} \rightarrow B_{r_{0}}^{\beta}$. Thus, we have that the set $B_{r_{0}}^{\beta}$ is relatively compact in $H_{\alpha}[0,1]$ for any $0<\alpha<\beta \leq 1$. Furthermore, $B_{r_{0}}^{\beta}$ is a compact subset in $H_{\alpha}[0,1]$.

We will show that the operator $F$ is continuous on $B_{r_{0}}^{\beta}$ with respect to the norm $\|\cdot\|_{\alpha}$, where $0<\alpha<\beta \leq 1$. Let $y \in B_{r_{0}}^{\beta}$ be an arbitrary point in $B_{r_{0}}^{\beta}$. Then, we get

$$
\begin{aligned}
(F x)(t)-(F y)(t)-((F x)(s)-(F y)(s))= & \left(T_{1} x\right)(t)+\left(T_{2} x\right)(t) \int_{0}^{1} k(t, \tau)\left(T_{3} x\right)(\tau) d \tau \\
& -\left(T_{1} y\right)(t)-\left(T_{2} y\right)(t) \int_{0}^{1} k(t, \tau)\left(T_{3} y\right)(\tau) d \tau \\
& -\left(T_{1} x\right)(s)-\left(T_{2} x\right)(s) \int_{0}^{1} k(s, \tau)\left(T_{3} x\right)(\tau) d \tau \\
& +\left(T_{1} y\right)(s)+\left(T_{2} y\right)(s) \int_{0}^{1} k(s, \tau)\left(T_{3} y\right)(\tau) d \tau
\end{aligned}
$$

for any $x \in B_{r_{0}}^{\beta}$ and $t, s \in[0,1]$. The equality (3.5) can be rewritten as:

$$
\begin{aligned}
(F x)(t)-(F y)(t)-((F x)(s)-(F y)(s))= & \left(T_{1} x\right)(t)-\left(T_{1} y\right)(t)-\left(\left(T_{1} x\right)(s)-\left(T_{1} y\right)(s)\right) \\
& +\left(T_{2} x\right)(t) \int_{0}^{1} k(t, \tau)\left(T_{3} x\right)(\tau) d \tau-\left(T_{2} y\right)(t) \int_{0}^{1} k(t, \tau)\left(T_{3} x\right)(\tau) d \tau \\
& +\left(T_{2} y\right)(t) \int_{0}^{1} k(t, \tau)\left(T_{3} x\right)(\tau) d \tau-\left(T_{2} y\right)(t) \int_{0}^{1} k(t, \tau)\left(T_{3} y\right)(\tau) d \tau \\
& -\left(T_{2} x\right)(s) \int_{0}^{1} k(s, \tau)\left(T_{3} x\right)(\tau) d \tau+\left(T_{2} y\right)(s) \int_{0}^{1} k(s, \tau)\left(T_{3} x\right)(\tau) d \tau \\
& -\left(T_{2} y\right)(s) \int_{0}^{1} k(s, \tau)\left(T_{3} x\right)(\tau) d \tau+\left(T_{2} y\right)(s) \int_{0}^{1} k(s, \tau)\left(T_{3} y\right)(\tau) d \tau .
\end{aligned}
$$

By (3.6), we have

$$
\begin{aligned}
(F x)(t)-(F y)(t)-((F x)(s)-(F y)(s))= & \left(T_{1} x\right)(t)-\left(T_{1} y\right)(t)-\left(\left(T_{1} x\right)(s)-\left(T_{1} y\right)(s)\right) \\
& +\left(\left(T_{2} x\right)(t)-\left(T_{2} y\right)(t)\right) \int_{0}^{1} k(t, \tau)\left(T_{3} x\right)(\tau) d \tau \\
& +\left(T_{2} y\right)(t) \int_{0}^{1} k(t, \tau)\left(\left(T_{3} x\right)(\tau)-\left(T_{3} y\right)(\tau)\right) d \tau \\
& -\left(\left(T_{2} x\right)(s)-\left(T_{2} y\right)(s)\right) \int_{0}^{1} k(s, \tau)\left(T_{3} x\right)(\tau) d \tau \\
& -\left(T_{2} y\right)(s) \int_{0}^{1} k(s, \tau)\left(\left(T_{3} x\right)(\tau)-\left(T_{3} y\right)(\tau)\right) d \tau .
\end{aligned}
$$


(3.7) yields the following equality:

$$
\begin{aligned}
((F x)(t)-(F y)(t))-((F x)(s)-(F y)(s))= & \left(T_{1} x\right)(t)-\left(T_{1} y\right)(t)-\left(\left(T_{1} x\right)(s)-\left(T_{1} y\right)(s)\right) \\
& +\left[\left(\left(T_{2} x\right)(t)-\left(T_{2} y\right)(t)\right)-\left(\left(T_{2} x\right)(s)-\left(T_{2} y\right)(s)\right)\right] \int_{0}^{1} k(t, \tau)\left(T_{3} x\right)(\tau) d \tau \\
& +\left(\left(T_{2} x\right)(s)-\left(T_{2} y\right)(s)\right) \int_{0}^{1}(k(t, \tau)-k(s, \tau))\left(T_{3} x\right)(\tau) d \tau \\
& +\left(\left(T_{2} y\right)(t)-\left(T_{2} y\right)(s)\right) \int_{0}^{1} k(t, \tau)\left(\left(T_{3} x\right)(\tau)-\left(T_{3} y\right)(\tau)\right) d \tau \\
& +\left(T_{2} y\right)(s) \int_{0}^{1}(k(t, \tau)-k(s, \tau))\left(\left(T_{3} x\right)(\tau)-\left(T_{3} y\right)(\tau)\right) d \tau .
\end{aligned}
$$

Since $\left|\left(T_{3} x\right)(\tau)\right| \leq\left\|T_{3} x\right\|_{\infty} \leq f_{3}\left(\|x\|_{\beta}\right)$ and $\left|\left(T_{3} x\right)(\tau)-\left(T_{3} y\right)(\tau)\right| \leq\left\|T_{3} x-T_{3} y\right\|_{\infty}$ for all $x, y \in B_{r_{0}}^{\beta}$ and $\tau \in[0,1]$, taking into account (3.8) and hypotheses, we can write:

$$
\begin{aligned}
\frac{|(F x)(t)-(F y)(t)-((F x)(s)-(F y)(s))|}{|t-s|^{\alpha}} \leq & \frac{\left|\left(T_{1} x\right)(t)-\left(T_{1} y\right)(t)-\left(\left(T_{1} x\right)(s)-\left(T_{1} y\right)(s)\right)\right|}{|t-s|^{\alpha}} \\
& +\frac{\left|\left(T_{2} x\right)(t)-\left(T_{2} y\right)(t)-\left(\left(T_{2} x\right)(s)-\left(T_{2} y\right)(s)\right)\right|}{|t-s|^{\alpha}} \int_{0}^{1}\left|k(t, \tau) \|\left(T_{3} x\right)(\tau)\right| d \tau \\
& +\frac{\left|\left(T_{2} x\right)(s)-\left(T_{2} y\right)(s)\right|}{|t-s|^{\alpha}} \int_{0}^{1}\left|k(t, \tau)-k(s, \tau) \|\left(T_{3} x\right)(\tau)\right| d \tau \\
& +\frac{\left|\left(T_{2} y\right)(t)-\left(T_{2} y\right)(s)\right|}{|t-s|^{\alpha}} \int_{0}^{1}\left|k(t, \tau) \|\left(T_{3} x\right)(\tau)-\left(T_{3} y\right)(\tau)\right| d \tau \\
& +\frac{\left|\left(T_{2} y\right)(s)\right|}{|t-s|^{\alpha} \int_{0}^{1}\left|k(t, \tau)-k(s, \tau) \|\left(T_{3} x\right)(\tau)-\left(T_{3} y\right)(\tau)\right| d \tau} \\
\leq & \left\|T_{1} x-T_{1} y\right\|_{\alpha}+\left\|T_{2} x-T_{2} y\right\|_{\alpha}\left\|T_{3} x\right\|_{\infty} K+\left\|T_{2} x-T_{2} y\right\|_{\infty}\left\|T_{3} x\right\|_{\infty} \int_{0}^{1} k_{\beta}|t-s|^{\beta-\alpha} d \tau \\
& +\left\|T_{2} y\right\|_{\alpha}\left\|T_{3} x-T_{3} y\right\|_{\infty} K+\left\|T_{2} y\right\|_{\infty}\left\|T_{3} x-T_{3} y\right\|_{\infty} \int_{0}^{1} k_{\beta}|t-s|^{\beta-\alpha} d \tau \\
\leq & \left\|T_{1} x-T_{1} y\right\|_{\alpha}+K\left\|T_{2} x-T_{2} y\right\|_{\alpha}\left\|T_{3} x\right\|_{\infty}+k_{\beta}\left\|T_{2} x-T_{2} y\right\|_{\alpha}\left\|T_{3} x\right\|_{\infty} \\
& +K\left\|T_{2} y\right\|_{\alpha}\left\|T_{3} x-T_{3} y\right\|_{\infty}+k_{\beta}\left\|T_{2} y\right\|_{\alpha}\left\|T_{3} x-T_{3} y\right\|_{\infty} \\
= & \left\|T_{1} x-T_{1} y\right\|_{\alpha}+\left(K+k_{\beta}\right)\left\|T_{2} x-T_{2} y\right\|_{\alpha}\left\|T_{3} x\right\|_{\infty}+\left(K+k_{\beta}\right)\left\|T_{2} y\right\|_{\alpha}\left\|T_{3} x-T_{3} y\right\|\left(_{\infty} .9\right)
\end{aligned}
$$

for all $t, s \in[0,1]$ with $t \neq s$. Besides, for $x, y \in B_{r_{0}}^{\beta}$, we obtain following equality:

$$
\begin{aligned}
(F x)(0)-(F y)(0)= & \left(T_{1} x\right)(0)+\left(T_{2} x\right)(0) \int_{0}^{1} k(0, \tau)\left(T_{3} x\right)(\tau) d \tau-\left(T_{1} y\right)(0)-\left(T_{2} y\right)(0) \int_{0}^{1} k(0, \tau)\left(T_{3} y\right)(\tau) d \tau \\
= & \left(T_{1} x\right)(0)-\left(T_{1} y\right)(0)+\left(T_{2} x\right)(0) \int_{0}^{1} k(0, \tau)\left(T_{3} x\right)(\tau) d \tau \\
& -\left(T_{2} y\right)(0) \int_{0}^{1} k(0, \tau)\left(T_{3} x\right)(\tau) d \tau+\left(T_{2} y\right)(0) \int_{0}^{1} k(0, \tau)\left(T_{3} x\right)(\tau) d \tau \\
& -\left(T_{2} y\right)(0) \int_{0}^{1} k(0, \tau)\left(T_{3} y\right)(\tau) d \tau \\
= & \left(T_{1} x\right)(0)-\left(T_{1} y\right)(0)+\left(\left(T_{2} x\right)(0)-\left(T_{2} y\right)(0)\right) \int_{0}^{1} k(0, \tau)\left(T_{3} x\right)(\tau) d \tau \\
& +\left(T_{2} y\right)(0) \int_{0}^{1} k(0, \tau)\left(\left(T_{3} x\right)(\tau)-\left(T_{3} y\right)(\tau)\right) d \tau .
\end{aligned}
$$


By (3.10), we get that

$$
\begin{aligned}
|(F x)(0)-(F y)(0)|= & \left|\left(T_{1} x\right)(0)-\left(T_{1} y\right)(0)\right|+\left|\left(T_{2} x\right)(0)-\left(T_{2} y\right)(0)\right| K \int_{0}^{1}\left|\left(T_{3} x\right)(\tau)\right| d \tau \\
& +\left|\left(T_{2} y\right)(0)\right| K \int_{0}^{1}\left|\left(T_{3} x\right)(\tau)-\left(T_{3} y\right)(\tau)\right| d \tau \\
\leq & \left\|T_{1} x-T_{1} y\right\|_{\infty}+\left\|T_{2} x-T_{2} y\right\|_{\infty} K\left\|T_{3} x\right\|_{\infty}+\left\|T_{2} y\right\|_{\infty} K\left\|T_{3} x-T_{3} y\right\|_{\infty} \\
\leq & \left\|T_{1} x-T_{1} y\right\|_{\alpha}+\left\|T_{2} x-T_{2} y\right\|_{\alpha} K\left\|T_{3} x\right\|_{\infty}+\left\|T_{2} y\right\|_{\alpha} K\left\|T_{3} x-T_{3} y\right\|_{\infty} .
\end{aligned}
$$

From (3.9) and (3.11), we have that

$$
\begin{aligned}
\|F x-F y\|_{\alpha} & =|(F x-F y)(0)|+H_{F x-F y}^{\alpha} \\
& =|(F x)(0)-(F y)(0)|+\sup \left\{\frac{|(F x)(t)-(F y)(t)-((F x)(s)-(F y)(s))|}{|t-s|^{\alpha}}: t, s \in[0,1] \text { and } t \neq s\right\} \\
& \leq 2\left\|T_{1} x-T_{1} y\right\|_{\alpha}+\left(2 K+k_{\beta}\right)\left\|T_{2} x-T_{2} y\right\|_{\alpha}\left\|T_{3} x\right\|_{\infty}+\left(2 K+k_{\beta}\right)\left\|T_{2} y\right\|_{\alpha}\left\|T_{3} x-T_{3} y\right\|_{\infty} \\
& \leq 2\left\|T_{1} x-T_{1} y\right\|_{\alpha}+\left(2 K+k_{\beta}\right)\left\|T_{2} x-T_{2} y\right\|_{\alpha}\left\|T_{3} x\right\|_{\infty}+\left(2 K+k_{\beta}\right)\left\|T_{2} y\right\|_{\beta}\left\|T_{3} x-T_{3} y\right\|_{\infty} \\
& \leq 2\left\|T_{1} x-T_{1} y\right\|_{\alpha}+\left(2 K+k_{\beta}\right)\left\|T_{2} x-T_{2} y\right\|_{\alpha} f_{3}\left(\|x\|_{\beta}\right)+\left(2 K+k_{\beta}\right) f_{2}\left(\|y\|_{\beta}\right)\left\|T_{3} x-T_{3} y\right\|_{\infty} .
\end{aligned}
$$

Moreover, since $\|x\|_{\beta} \leq r_{0}$ and $\|y\|_{\beta} \leq r_{0}$, we derive from (3.12) that the following inequality holds:

$$
\|F x-F y\|_{\alpha} \leq 2\left\|T_{1} x-T_{1} y\right\|_{\alpha}+\left(2 K+k_{\beta}\right) f_{3}\left(r_{0}\right)\left\|T_{2} x-T_{2} y\right\|_{\alpha}+\left(2 K+k_{\beta}\right) f_{2}\left(r_{0}\right)\left\|T_{3} x-T_{3} y\right\|_{\infty} .
$$

Since the operators $T_{1}, T_{2}: H_{\beta}[0,1] \rightarrow H_{\beta}[0,1]$ and $T_{3}: H_{\beta}[0,1] \rightarrow C[0,1]$ are continuous on $H_{\beta}[0,1]$ with respect to the norm $\|\cdot\|_{\alpha}$, they are also continuous at the point $y \in B_{r_{0}}^{\beta}$. Let us take an arbitrary $\varepsilon>0$, then there exists the number $\delta=\delta(\varepsilon)>0$. The inequalities

$$
\left\|T_{1} x-T_{1} y\right\|_{\alpha}<\frac{\varepsilon}{6},\left\|T_{2} x-T_{2} y\right\|_{\alpha}<\frac{\varepsilon}{3\left(2 K+k_{\beta}\right) f_{3}\left(r_{0}\right)}
$$

and

$$
\left\|T_{3} x-T_{3} y\right\|_{\infty}<\frac{\varepsilon}{3\left(2 K+k_{\beta}\right) f_{2}\left(r_{0}\right)}
$$

hold for all $x \in B_{r_{0}}^{\beta}$. Then, taking into account (3.13), we derive the following inequality:

$$
\|F x-F y\|_{\alpha}<\frac{\varepsilon}{3}+\frac{\varepsilon}{3}+\frac{\varepsilon}{3}=\varepsilon .
$$

for all $x \in B_{r_{0}}^{\beta}$ with $\|x-y\|_{\alpha}<\delta$. Eventually, we infer that the operator $F$ is continuous at the point $y \in B_{r_{0}}^{\beta}$. Since $y$ was chosen arbitrarily, we conclude that $F$ is continuous on $B_{r_{0}}^{\beta}$ with respect to the norm $\|\cdot\|_{\alpha}$. Because $B_{r_{0}}^{\beta}$ is compact in $H_{\alpha}[0,1]$, by the classical Schauder fixed point theorem, we get the desired consequence.

\section{Conclusion}

This article concerns the entity of solutions of the following a quadratic integral equation of Fredholm type,

$$
x(t)=\left(T_{1} x\right)(t)+\left(T_{2} x\right)(t) \int_{0}^{1} k(t, \tau)\left(T_{3} x\right)(\tau) d \tau, t \in I=[0,1] .
$$

where $k$ is given function, $T_{1}, T_{2}, T_{3}$ are given operators satisfying conditions specified later and $x$ is unknown function.

\section{References}

[1] M. Benchohra, M. A. Darwish, On unique Solvability of Quadratic Integral Equations with Linear Modification of the Argument, Miskolc Math. Notes, 10 (2009), 3-10.

[2] J. Banaś, R. Nalepa, On the space of functions with growths tempered by a modulus of continuity and its applications, J. Funct. Space Appl. (2013), 13 pages, doi:http://dx.doi.org/10.1155/2013/820437. 
[3] J. Caballero, M. Abdalla, K. Sadarangani, Solvability of a quadratic integral equation of fredholm type in Hölder spaces, Electron. J. Differ. Eq., 31 (2014), 1-10.

[4] J. Schauder, Der Fixpunktsatz in Funktionalriiumen, Studia Math., 2 (1930), 171-180.

[5] J. Banaś, A. Chlebowicz, On an elementrary inequality and its application in theory of integral equations, J. Math. Ineq., 11 (2) (2017), 595-605.

[6] J. Caballero, B. López, K. Sadarangani, Existence of nondecreasing and continuous solutions of an integral equation with linear modification of the argument, Acta Math. Sin. (Engl. Ser.), 23 (9) (2007), 1719-1728.

[7] M. A. Darwish, On quadratic integral equation of fractional orders, J. Math. Anal. Appl., 311(1) (2005),112-119.

[8] S. Hu, M. Khavanin, W. Zhuang, Integral equations arising in the kinetic theory of gases, Appl. Anal., 34 (1989),261-266.

[9] C. A. Stuart, Existence theorems for a class of non-linear integral equations, Math. Z., 137 (1974) $49-66$.

[10] J. Banaś, A. Chlebowicz, On existence of integrable solutions of a functional integral equation under Carathéodory conditions, Nonlinear Anal., 70 (2009), 3172-3179.

[11] J. Banaś, B. Rzepka, On local attractivity and asymptotic stability of solutions of a quadratic Volterra integral equation, Appl. Math. Comput., 213 (2009), 102-111.

[12] H. Deepmala, K. Pathak, A study on some problems on existence of solutions for nonlinear functional-integral equations, Acta Math. Scientia, 33 (2013), 1305-1313.

[13] H. Deepmala, K. Pathak, Study on existence of solutions for some nonlinear functional-integral equations with applications, Math. Commun., 18 (2013), 97-107.

[14] K. Maleknejad, K. Nouri, R. Mollapourasl, Existence of solutions for some nonlinear integral equations, Communications in Commun. Nonlinear Sci. Numer. Simul., 14 (2009), 2559-2564.

[15] K. Maleknejad, K. Nouri, R. Mollapourasl, Investigation on the existence of solutions for some nonlinear functional-integral equations, Nonlinear Anal., 71 (2009), 1575-1578.

[16] L. N. Mishra, R. P. Agarwal, M. Sen, Solvability and asymptotic behavior for some nonlinear quadratic integral equation involving Erdélyi-Kober fractional integrals on the unbounded interval, Prog. Frac. Differ. Appli., 2 (3) (2016).

[17] L. N. Mishra, M. Sen, On the concept of existence and local attractivity of solutions for some quadratic Volterra integral equation of fractional order, Appl. Math. Comput., (2016), http://dx.doi.org/10.1016/j.amc.2016.03.002.

[18] L. Liu, F. Guo, C. Wu, Y. Wu, Existence theorems of global solutions for nonlinear Volterra type integral equations in Banach spaces, J. Math. Anal. Appl., 309 (2005), 638-649.

[19] J. Mallet-Paret, R.D. Nussbam, Inequivalent measures of noncompactness and the radius of the essential spectrum, Proc. Amer. Math. Soc., (2010), 917-930.

[20] G. Micula , G. Fairweather, Direct numerical spline methods for first order Fredholm integro-differential equations, Rev. Anal. Numer. Theory Approx., 22 (1) (1993), 59-66.

[21] M. Mursaleen, S.A. Mohiuddine, Applications of noncompactness to the infinite system of differential equations in $\ell_{p}$ spaces, Nonlinear Anal. Theory Methods Appl., 75 (4) (2012), 2111-2115.

[22] L. Olszowy, Solvability of infinite systems of singular integral equations in Fréchet space of continuous functions, Comp. Math. Appl. 59 (2010), 2794-2801.

[23] M. J. Caballero, R.Nalepa, K. Sadarangani, Solvability of a quadratic integral equation of Fredholm type with Supremum in Hölder Spaces, J. Funct. Space Appl., (2014), 7 pages, doi:http://dx.doi.org/10.1155/2014/856183. 